\title{
Fabric defect detection based on wavelet transform and k-means Zhang Huanuan $^{\mathrm{a}}$, Zhao Juan ${ }^{\mathrm{b}}$, Li Renzhong ${ }^{\mathrm{c}}$, Jing Junfeng ${ }^{\mathrm{d}}$, Li Pengfei ${ }^{\mathrm{e}}$ \\ School of Electronic and Information, Xi'an Polytechnic University, Xi'an, Shaanxi, 710048, China

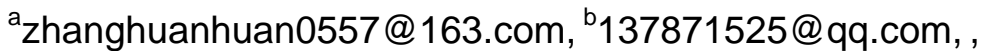 ‘649674796@qq.com, d413066458@qq.com, ${ }^{\mathrm{l}} \mathrm{li6208@163.com}$
}

Keywords: Wavelet Transform; k-means; Fabric Defect

\begin{abstract}
A novel method based on wavelet transform and k-means is proposed to solve the problem of automated fabric defect detection which is more essential and important in assuring the fabric quality. Firstly, the fabric images are processed using wavelet decomposition algorithm, and the produced wavelet coefficients are used to restructure the new images using wavelet thresholding denoising. Secondly, the obtained new images are segmented by applying the k-means algorithm. The results demonstrate that the proposed scheme is able to efficiently locate the position of fabric defect and segment the defect.
\end{abstract}

\section{Introduction}

Fabric quality detection plays an important role in the textile industry, and the emergence of fabric flaw in a large degree affects the quality of the fabric, but also affects the cost of textile enterprises. According to some studies, the defects of fabrics may reduce the price of a product by 45\%-65\%[1].

Fabric defect detection methods are backward for many domestic enterprises at present. The accuracy rate of fabric defect detection is low. In addition, it is a great challenge to detect accurately fabric defects which own to a wide range and varieties of shapes of fabric defects. It is urgent to develop more advanced and more intelligent defect detection methods to adopt the needs of modern enterprise production, and to solve the problem of the slow speed, low accuracy and other shortcomings of traditional defect detection methods, to improve corporate profits. The automatic defect detection research reflected the significant and commercial value.

In recent years, many domestic and foreign researches on fabric defect detection have been done and have achieved certain results. Bu et.al.[2] presented the multi-fractal image features and support vector data description for fabric defect detection. Ngan et.al.[3] used the rule with fabric defect detection method. Muller et.al.[4] adopted two forms of the fabric filter successfully to identify flaws. Mak et.al.[5] used morphological filter to complete fabric defect detection. Tsai and $\mathrm{Hu}[6]$ proposed four different fabric defects Fourier model and used these models to extract Fourier features of fabric defects. Ozdemir and Ercil [7] used Gauss Markov random fields to detect common fabric defect. Wong et al[8]. proposed wavelet transform and BP neural network to detect the fabric defect. Although existed methods may detect fabric defects, it is limited to detect the fabric defects due to a wide range and varieties of shapes of fabric defects, how to effectively detected is still difficult and deserve to research.

We propose an effective method of segmenting defects with a relatively consistent background texture based on based on wavelet transform and k-means. The experimental results showed that the proposed method had excellent defect detection performance which can detect the different types of defects and different types of defects and different texture.

\section{Wavelet transform}

Wavelet transform can effectively describe the fabric texture feature. Mallat applied two one-dimensional convolution algorithm to achieve the fast wavelet decomposition in two-dimensional and to extract the texture features using the decomposition of images. 
Wavelet transform of image size of $M \times N$ image $f(x, y)$ is defined as follows:

$$
\begin{gathered}
W_{u}(0, m, n)=\frac{1}{\sqrt{M N}} \sum_{x=0}^{M-1} \sum_{y=0}^{N-1} f(x, y) u_{0, m, n}(x, y) \\
W_{v}^{(i)}(j, m, n)=\frac{1}{\sqrt{M N}} \sum_{x=0}^{M-1} \sum_{y=0}^{N-1} f(x, y) v_{0, m, n}^{(i)}(x, y),(i)=\{H, V, D\}
\end{gathered}
$$

Where,

$$
\begin{array}{r}
u_{j, m, n}(x, y)=2^{j / 2} u\left(2^{j} x-m, 2^{j} y-n\right) \\
v_{j, m, n}^{(i)}(x, y)=2^{j / 2} v^{(i)}\left(2^{j} x-m, 2^{j} y-n\right),(i)=\{H, V, D\}
\end{array}
$$

$M=N=2^{J}, j=0,1,2 \ldots, J-1, \quad m, n=0,1,2, \ldots, 2^{j}-1 . v^{H,} v^{V,} v^{D}$ are three directional sensitivity of wavelet functions in horizontal, vertical, and diagonal, respectively. $u(x, y)$ is separable scaling function.

Figure 1 (a)presents the wavelet decomposition procedure. $L_{o}$ and $H_{i}$ represent low-pass filter, high-pass filter, respectively and $\downarrow 2$ is down-sampling in 2 even point. The step of wavelet decomposition as follows: In the first place, the two-dimensional fabric image is divided into a series of row vector and column vector. Then, we obtained the corresponding high frequency matrix by sampling the high frequency signals and low frequency signal and retaining the subscript of row vector in even number elements. The low frequency signal obtains corresponding to low frequency matrix. The new generation of the rows matrix vector is used to filter and down-sample. Through processing above step , we can obtain a low and three high frequency signals.

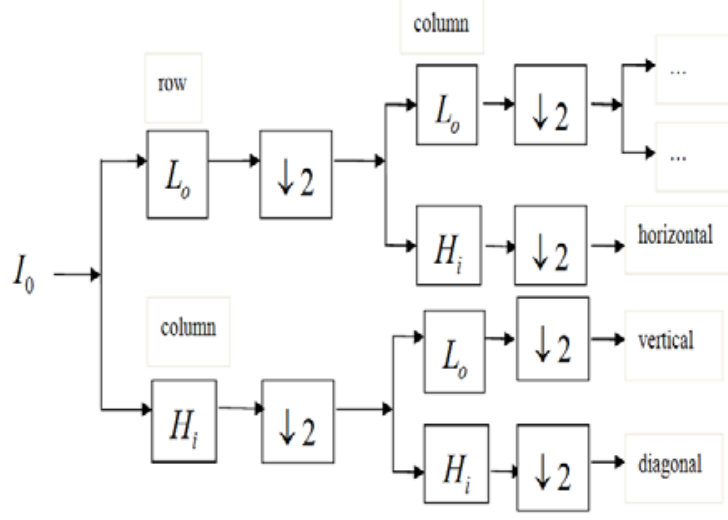

(a)

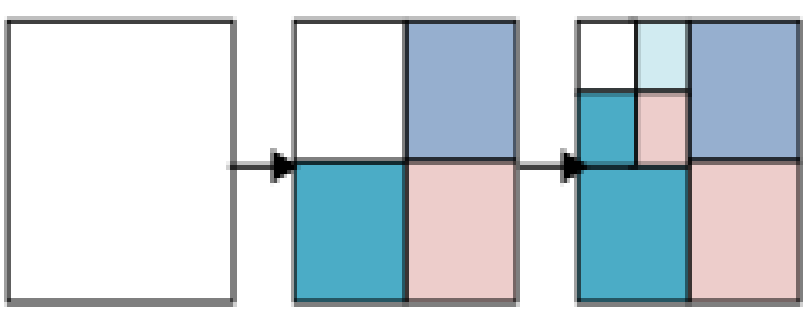

(b)

Figure 1 The process of wavelet decomposition

The generating coefficients of wavelet decomposition is used to reconstruct a new image. In this paper we use the wavelet threshold denoising to process the wavelet decomposition coefficients, and obtain the denoising image via reconstructing the wavelet decomposition coefficients. In the denoising process, threshold function reflected different processing strategies and different estimation methods about the wavelet decomposition coefficients. Threshold denoising expression as follows:

$$
w_{\lambda}= \begin{cases}{[\operatorname{sig}(w)](|w|-\lambda),} & |w| \geq \lambda \\ 0, & |w|<\lambda\end{cases}
$$

Figure 2 shows wavelet soft-threshold denoising method for fabric defect sample . 

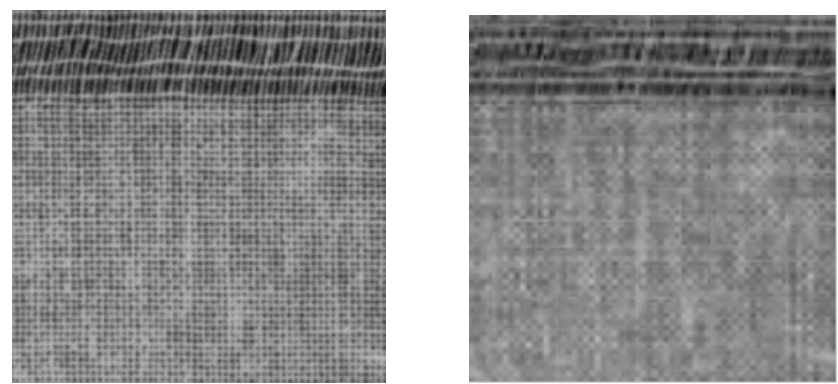

Figure 2 The wavelet soft-threshold denoising for sample

\section{k-means Algorithm}

$k$-means algorithm widely used in pattern recognition and clustering. The core idea of k-means algorithm is to select $k$ initial cluster centers and divide the data into $k$ categories through an iterative process, then making the evaluation clustering performance criterion function to achieve optimal, so that each cluster generated compact between class independence. The sample data of the same data type is divided according to minimum rules to get each equivalence class. In this paper, we used squared error criterion function as formula (6).

$$
J_{c}(m)=\sum_{j=1}^{k} \sum_{X_{i} \in C_{j}}\left|X_{i}-Z_{j}\right|^{2}
$$

Where, $J_{c(m)}$ is the sum of squared errors of all objects in the database, $X_{i}$ represents the given data object, $Z_{j}$ is the average of the cluster $C_{j}$.

The specific steps of $k$-means clustering algorithm can be accomplished:

Firstly, determining an initial cluster center for each cluster, so that you can get the $k$ initial cluster centers. secondly, a sample set of samples are assigned to the nearest cluster following the principle of the minimum distance, then, using each cluster of the sample mean as a new cluster centers. finally, repeat the previous two steps until the cluster center does not change. It can be obtained after the end of the $k$ clusters.

In this paper, gray pixels of the fabric image are clustered using the characteristics of $k$-means clustering algorithm to judge fabric image pixel which belongs to the category of defects or non-defect categories.

\section{Test results}

Fabric defect is looked as a special texture and normal fabric is looked as another texture. Fabric defect can be seen as a special texture, while the normal fabric can be seen as another texture. That is to say, fabric defect is a class and the normal fabric is another class. According to this feature, we propose a method based on wavelet decomposition and k-means algorithm to detect fabric defects. To begin with, the sample images are processed using wavelet decomposition algorithm, the produced wavelet coefficients were used to restructure the new images using wavelet thresholding denoising. Then the restructured new image is used in k-means clustering input to compute the sample image pix belong to defect or normal fabric. Finally, the final cluster results are processed by binarization to segment the fabric defect. In this paper, the experimental fabric samples are from the TILDA database, and the sample size is $256 \times 256$. Parts of experiment results shown in Figure 3 , Figure 3 (a) fabric defects image, Figure 3 ((b) is reconstructed a new image using the generating coefficients of wavelet decomposition, Figure 3 (c)is the test results, Figure 3(d) is Gaussian Mixture Model (GMM ) test results, where white is the defect area, black is the normal fabric area. Experiments shown that our proposed method is more effective than GMM method. 


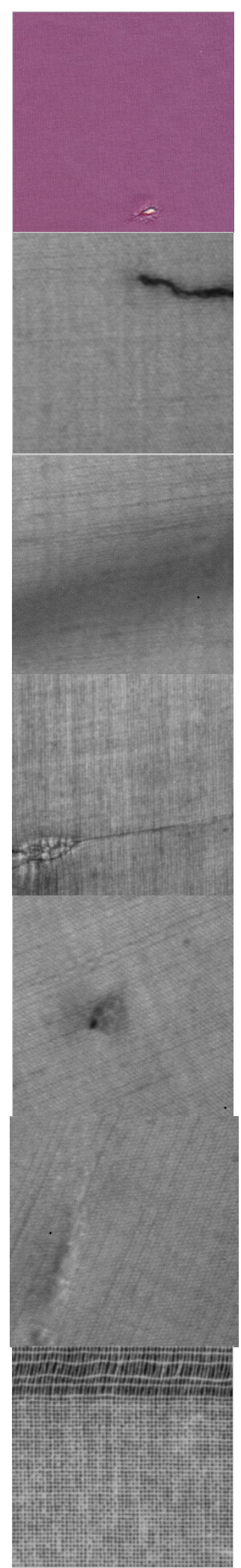

(a)

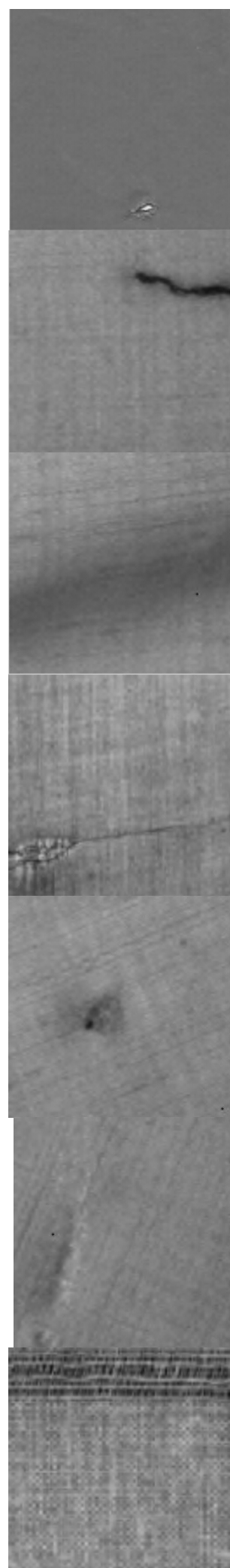

(b)
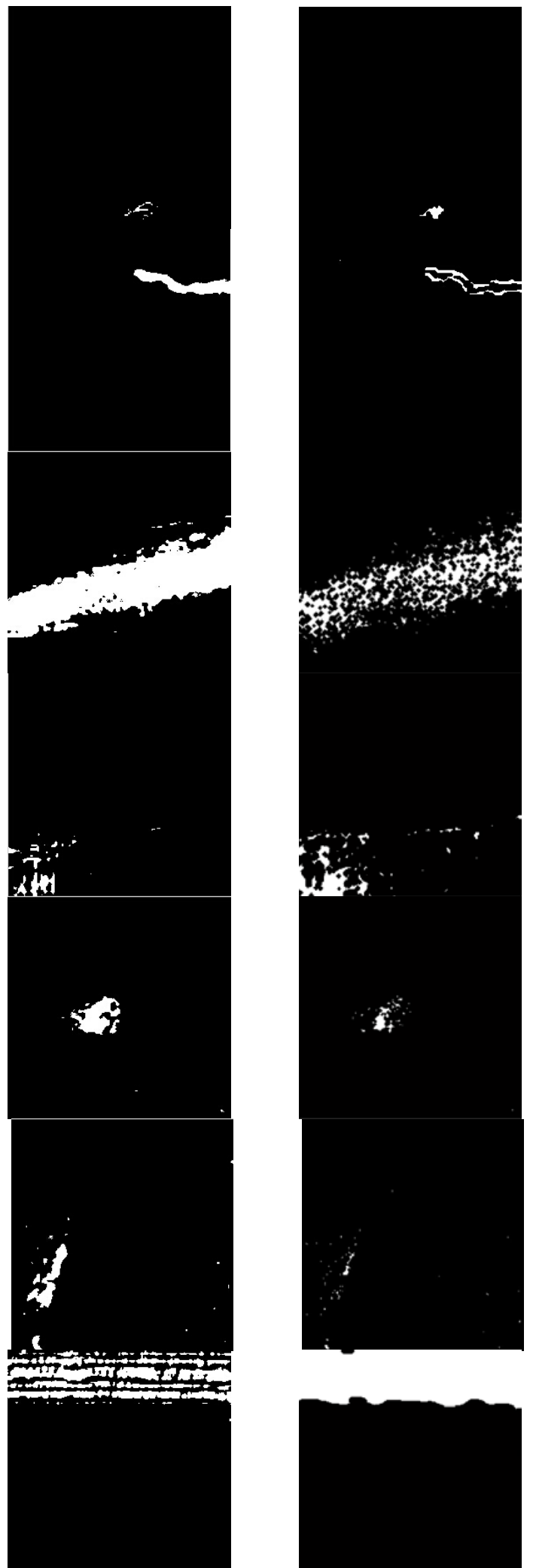

(c)

(d)

Figure 3 parts of defects segmentation results

(a) fabric defects image (b) reconstruct a new image using the generating coefficients of wavelet decomposition (c) our proposed the test results (d) Gaussian Mixture Model test results

\section{Conclusion}

An algorithm of faric defect detection based on wavelet decomposition and k-means has been presented. In the first step, the sample image is processed by wavelet decomposition algorithm, then, 
the produced wavelet coefficients are to reconstruct the new image via wavelet thresholding denoising. In the second step, the new images obtained are divided into parts: the no-defect and the defect using k-means. The detection results showed that the efficiency and effectiveness of the proposed approach could be applied to detect and locate fabric defects.

\section{Acknowledgement}

In this paper, the research was sponsored by the Natural Science Foundation of China (61301276), Shaanxi Provincial Education Department (Program No. 2013JK1084), China National Textile And Apparel Council (Program No.2013066) and Undergraduate Training Programs for Innovation and Entrepreneurship of Xi'an Polytechnic University(Program No.201303012).

\section{References}

[1] Zhang Y. H \& Wong W.K. An Intelligent model for detecting and classifying color-textured fabric defects using genetic algorithms and the Elman neural network [J]. Textile Research Journal.2011, 81(17):1772-1787.

[2] Bu H G, Wang J, Huang X B. Fabric defect detection based on multiple fractal features and support vector data description [J]. Engineering Applications of Artificial Intelligence, 2009, 22 (2): 224-235.

[3] Ngan H Y T, Pang G K H. Regularity Analysis for Patterned Texture Inspection[J].Automation Science and Engineering, IEEE Transactions on, 2009, 6(1):131-144.

[4] Mueller S, Nickolay B. Morphological image processing for the recognition of surface defects[C].Optics for Productivity in Manufacturing. International Society for Optics and Photonics, 1994: 298-307.

[5] Mak K L, Peng P, Yiu K F C. Fabric defect detection using morphological filters. Image and Vision Computing [J]. 2009, 27 :1585-1592.

[6] Tsai I S, Hu M C. Automatic inspection of fabric defects using an artificial neural network technique[J]. Textile Research Journal, 1996, 66(7): 474-482.

[7] S. Ozdemir, A. Ercil. Markov random fields and Karhumen-Loeve transforms for defect inspection of textile products[J]. in: Proceedings of the IEEE Conference on Emerging Technologies and Factory Automation, 1996, 2:697-703.

[8] Wong W. K, Yuen C. W. M, Fan D. D, Chan L. K \& Fung E. H. K. Stitching defect detection and classification using wavelet transform and BP neural network[J]. Expert Systems with Applications. 2009, 36: 3845-3856. 